\title{
CORRELAÇÃO ENTRE DISTÚRBIOS DO SONO E AVALIAÇÃO COGNITIVA EM ESTUDANTES DE MEDICINA
}

\author{
Átila Mourão Lima* \\ Julia Rodrigues Marcondes Dutra* \\ Julio César Tolentino Junior \\ Arthur Fernandez Cortez $z^{\text {*t* }}$ \\ Roberta Benitez Freitas Passos \\ Wagner Martignoni de Figueiredo
}

\begin{abstract}
RESUMO: Estudantes de medicina compõem um grupo suscetível aos transtornos do sono e suas consequências, como disfunção de capacidades cognitivas. Este estudo, transversal e descritivo, tem como objetivo principal analisar 0 sono dos estudantes de medicina e sua correlação com alterações cognitivas por meio de testes neuropsicológicos. Foram incluídos aleatoriamente 50 alunos de graduação em medicina da Universidade Federal do Estado do Rio de Janeiro (Unirio). Utilizaram-se os seguintes instrumentos de avaliação: Índice de Qualidade de Sono de Pittsburgh (PSQI); Escala de Sonolência de Epworth (ESE); análise da presença de insônia e seus subtipos; Trail Making Test (TMT) Parte A e Parte B; Digit Symbol Substitution Test (DSST). Dos 50 estudantes avaliados, 27 (54\%) eram do sexo feminino e 23 (46\%), do masculino. A idade variou de 19 e 32 anos (22,9 \pm 2,9 anos). 0 tempo médio de sono foi de aproximadamente $6 \mathrm{~h}$. Trinta e oito (76\%) alunos relataram sintomas de insônia, 37 (74\%) apresentaram "qualidade rum do sono", de acordo com o PSQl, e 16 (32\%) preenchiam pontuação na ESE para sonolência excessiva diurna (SED). Diferente do esperado, não houve correlação entre a presença de sintomas de insônia, menor tempo de sono noturno, pior qualidade de sono e critérios para SED com pior desempenho no TMT e DSST em estudantes de medicina.
\end{abstract}

PALAVRAS-CHAVE: Insônia; Cognição; Sonolência Excessiva Diurna.

\section{CO-RELATION BETWEEN SLEEP DISORDERS AND COGNITIVE ASSESSMENT OF STUDENTS OF MEDICINE}

\begin{abstract}
University students of Medicine are susceptible to sleep disorders and their consequences, such as dysfunction of their cognitive abilities. Current study analyzes the sleep of students of Medicine by neuropsychological tests. Fifty undergraduate students in the Medicine Course of the Federal University of the State of Rio de Janeiro (UNIRIO) were included at random and the Pittsburgh Sleep Quality Index (PSQI), Eppworth's Sleepiness Scale, analysis of insomnia and its subtypes, and Trail Making Test (TMT) Parts A and B; Digit Symbol Substitution Test (DSST) were employed for assessment. Further, 27 (54\%) out of 50 students evaluated were

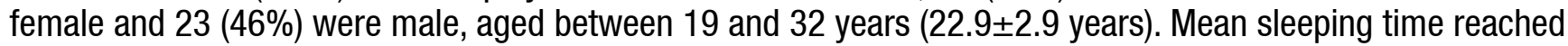
6 hours. Thirty-eight $(76 \%)$ students reported insomnia and thirty-seven $(74 \%)$ revealed bad sleeping quality according to PSQI and sixteen (32\%) scores EDE for excessive day sleeping bouts. Contrary to what was expected, there was no co-relationship between insomnia symptoms, less night sleeping time, bad sleeping quality and criteria for day sleeping bouts, with a worse performance for TMT and DSST in undergraduates of the Medicine Course.
\end{abstract}

KEYWORDS: Insomnia; Cognition; Excessive Day Sleeping.

\footnotetext{
"Discente do curso de medicina da Universidade Federal do Estado do Rio de Janeiro (UNIRI0), Rio de Janeiro, Brasil.

"Médico no Hospital Universitário Gaffrée e Guinle, Rio de Janeiro, Brasil.

** Mestre em Ciências Médicas pela Universidade do Estado do Rio de Janeiro (UERJ); Docente assistente na Universidade Federal do Estado do Rio de Janeiro (UNIRIO), Rio de Janeiro, Brasil; E-mail: juliotolentinonovo@gmail.com

*** Mestre em Ciências Médicas pela Universidade Federal do Rio de Janeiro (UFRJ); docente auxiliar da Universidade Federal do Estado do Rio de Janeiro (UNIRIO), Rio de Janeiro, Brasil.

${ }^{*}$ Doutora em Epidemiologia pelo Instituto de Medicina Social da UERJ; Docente adjunta da Universidade Federal do Estado do Rio de Janeiro (UNIRI0).

Mestre em Neurologia pela Universidade Federal Fluminense (UFF); Docente associado da Universidade Federal do Estado do Rio de Janeiro (UNIRI0).
} 


\section{INTRODUÇÃo}

0 sono pode ser definido como um estado de inconsciência do qual uma pessoa pode ser despertada por um estímulo sensorial ou outro estímulo (GUYTON; HALL, 2006), composto por duas fases: a que apresenta movimentos oculares rápidos, em inglês, rapid eyes movement (REM), e o sono de ondas lentas, em que este movimento não ocorre (CARVALHO et al., 2013). Apesar de ainda não serem bem compreendidos os papéis exatos de cada um dos estágios do sono ou qual a base fisiológica para a alternância de fases, a fase REM tem papel importante na consolidação da memória procedimental (knowing how), ao passo que 0 sono de ondas lentas seria responsável pela consolidação da memória declarativa (knowing what) (HERSHNER; CHERVIN, 2014).

Entre as consequências negativas da privação de sono, estão incluídos transtornos do humor, alterações cognitivas e na atividade motora. 0 prejuízo cognitivo ocorre por uma instabilidade do estado de vigília, acumulando-se enquanto o paciente estiver insone, e está relacionado a uma adaptação à sensação subjetiva de sonolência. No entanto a hipótese que correlaciona a falta de sono com 0 déficit das capacidades cognitivas ainda carece de maiores estudos para melhor elucidação do assunto (DURMER; DINGER, 2005).

Os domínios cognitivos mais prejudicados pela privação do sono são a atenção, a memória de trabalho, responsável pelo armazenamento e manejo temporário de informações para a realização de uma tarefa (ROZENTHAL, 2011), e a função executiva, domínio que organiza outras funções, como habilidade visuoespacial, memória e práxis, visando à formação do pensamento abstrato, procedendo deste para 0 planejamento de ações (DURMER; DINGER, 2005).

A sensação de sono insuficiente, ou não reparador, é uma das queixas mais prevalentes na população geral, porém raramente é valorizada pelo médico ou pelo próprio paciente. Com isso, é uma condição bastante subdiagnosticada e inadequadamente tratada. Dependendo dos critérios utilizados para esse diagnóstico (por exemplo, sintomas de insônia ou apenas insatisfação com 0 próprio sono), a sua prevalência pode variar de 5 a $50 \%$ em populações estudadas. No entanto, quando são utilizados critérios mais estritos para o diagnóstico, a sua prevalência cai, então, para 6 a 10\%(MORIN; JARRIN, 2013).

A sonolência diurna excessiva (SED), que frequentemente acompanha indivíduos com insônia, pode estar relacionada a prejuízo de domínios da cognição e com vários problemas sociais como absenteísmo, acidentes de trânsito e, finalmente, redução da capacidade de aprendizado de estudantes (HERSHNER; CHERVIN, 2014).

0 curso de medicina, por ser bastante demandante em termos acadêmico-práticos, com atividades curriculares em horário integral, engajamento em atividades extracurriculares, plantões noturnos, além da pressão e estresse a que se submetem pela exigência de alto rendimento e tempo despendido em estudos, acaba por prejudicar o sono do estudante, o que traz potencial prejuízo do aproveitamento durante a fase de graduação médica (CARDOSO et al., 2009). Como alterações no padrão do sono noturno e do diurno podem estar relacionadas a prejuízo de domínios da cognição, foi realizado estudo em estudantes de medicina com o objetivo de analisar a correlação entre a presença de transtornos do sono com o desempenho na execução de testes neuropsicológicos.

\section{METODOLOGIA}

0 estudo, de delineamento transversal $\mathrm{e}$ descritivo, foi realizado com alunos do curso de medicina da Universidade Federal do Estado do Rio de Janeiro (Unirio), escolhidos aleatoriamente, que compreenderam eassinaram otermo de consentimento livre e esclarecido. Foram excluídos aqueles que não preencheram por completo os questionários ou que optaram por não participar do estudo.

Instrumentos de avaliação foram aplicados 
por três examinadores durante 0 período letivo, para analisar transtornos do sono e correlacionar estes com alterações cognitivas por meio de testes neuropsicológicos.

Foi utilizado, além do protocolo com dados demográficos gerais dos alunos incluídos no estudo, 0 descrito abaixo:

- Índice de Qualidade de Sono de Pittsburgh (PSQI);

- Escala de Sonolência de Epworth (ESE);

- Ficha específica para análise da presença e tipo de insônia;

- Trail Making Test (TMT) Parte A;

- Trail Making Test (TMT) Parte B;

- Digit Symbol Substitution Test (DSST).

0 PSQI se refere à qualidade do sono no último mês, fornecendo um índice de gravidade e natureza do transtorno. É realizado por meio de 19 perguntas agrupadas em sete componentes do sono: qualidade subjetiva, latência, duração, eficiência habitual, distúrbios, uso de medicação e disfunções diurnas; cada um avaliado em uma escala de 0 a3, com o mesmo peso, em que 0 "três" reflete 0 extremo negativo da escala tipo Likert (BUYSSE; REYNOLDS; MONK, 1989). A soma dos valores desses componentes forma a pontuação final entre 0-21 pontos que constitui um índice: o PSQI Total. Pontuações de 0-4 indicam boa qualidade do sono e maiores ou iguais a 5 pontos indicam qualidade ruim de sono. Para se calcular os valores finais, foram utilizadas as instruções dos escores do PSQI ou Scoring Instructions for the Pittsburgh Sleep Quality Index (CHELLAPPA; ARAUJO, 2007).

Para se rastrear a presença de SED, utilizouse a Escala de Sonolência de Epworth (ESE), já testada e validada para língua portuguesa (BERTOLAZI et al., 2009). É um questionário que se refere à possibilidade de cochilar em oito situações cotidianas. Para graduar a probabilidade de cochilar, 0 indivíduo utiliza uma escala de 0 (zero) a 3 (três), em que 0 corresponde a nenhuma e 3 , à grande probabilidade de cochilar. Para definição de SED, é necessária uma pontuação total acima de 10 (BERTOLAZI et al., 2009).

$\mathrm{Na}$ ficha de alterações do sono, foram pesquisados se 0 aluno apresenta critérios para insônia e os tipos desse transtorno do sono (insônia inicial, intermediária, terminal ou sono não reparador).

0 TMT consiste em um instrumento manual, composto de duas etapas (partes A e B): TMT parte $A$, composto apenas por números, em que 0 indivíduo deverá desenhar com um lápis ou caneta uma linha que conecte em ordem crescente, em sequência numérica, a série de números (1 a 25) dispostos em círculos ao acaso em uma folha de papel. No TMT parte $\mathrm{B}, 0$ indivíduo deve alternar entre números $\mathrm{e}$ letras também sequencialmente (e.g.: 1, A, 2, B, 3, C), ligando os números de $1 \mathrm{a} 12$, alteradamente às letras de $A$ a $L$, dentro de círculos em sequências alternadas em ordem crescente. A correção do TMT é baseada no tempo (em segundos) utilizado para se concluir corretamente cada parte do teste. A pontuação em cada parte do teste é o tempo que a pessoa leva para terminá-lo. 0 TMT fornece informações sobre as habilidades de busca visual, scanning, velocidade de processamento, flexibilidade mental e funções executivas (TOMBAUGH, 2004).

ODSST é um teste neuropsicológico psicomotor em que o paciente, em posse de papel e caneta, tem a sua frente uma tabela com uma sequência de números e seus correspondentes em símbolos e deve, então, completar as lacunas vazias de outra tabela que possui apenas números. 0 tempo máximo para 0 término do teste é de $90 \mathrm{seg}$. A pontuação consiste no número de correlações corretas entre os números $\mathrm{e}$ os símbolos. 0 teste avalia principalmente habilidade visuoespacial, memória de trabalho, organização perceptual e atenção seletiva (LÓPEZ-GÓNGORA; QUEROL; ESCARTÍN, 2015).

Os resultados foram expressos em média, desvio padrão, mediana ou porcentagens. A distribuição das variáveis foi analisada por histograma e teste de Normalidade Shapiro-Wilk. Para a comparação entre as amostras independentes, foi utilizado $o$ teste $t$ de Student para as variáveis contínuas com distribuição normal ou o teste não paramétrico de Mann-Whitney 
no caso de variáveis contínuas sem distribuição normal.

0 coeficiente de correlação de Pearson foi utilizado para se analisar a correlação entre as variáveis quantitativas de distribuição normal.

Para a análise estatística foi utilizado o SPSS

\section{RESULTADOS}

Na Tabela 1 são apresentadas as principais características demográficas dos 50 (n) estudantes do curso de medicina da Unirio incluídos neste trabalho. $21 \circledR$.

Tabela 1. Descritiva das variáveis para a amostra geral

\begin{tabular}{lcccccc}
\hline \multicolumn{1}{c}{ Variável } & $\mathbf{n}$ & Média & Desvio padrão & Mediana & Mínimo & Máximo \\
\hline Idade (anos) & 50 & 22,9 & 2,9 & 23 & 19 & 32 \\
Horas de sono & 50 & 6,5 & 1,3 & 6,5 & 3 & 10 \\
PSQI (pontuação) & 50 & 6,7 & 2,8 & 6,5 & 2 & 15 \\
ESSE (pontuação) & 50 & 8,9 & 2,9 & 8,0 & 16 & 3,0 \\
TMT Parte A & 50 & 26,4 & 6,8 & 26,9 & 11 & 42 \\
TMT parte B & 50 & 43,5 & 17,3 & 40 & 120 & 22 \\
DSST & 50 & 55,7 & 7,8 & 58 & 37 & 63 \\
\hline
\end{tabular}

PSQI - Índice de Qualidade de Sono de Pittsburgh; ESE - Escala de Sonolência de Epworth; TMT - Trail Making Test; DSST - Digit Symbol Substitution Test.

A idade variou de 19 a 32 anos $(22,9 \pm 2,9$ anos), sendo 27 (54\%) estudantes do sexo feminino e 23 (46\%) do sexo masculino. Em relação ao período da faculdade dos alunos estudados, houve predomínio dos $4^{\circ}(24 \%), 5^{\circ}(32 \%)$ e $6^{\circ}(24 \%)$ períodos.
Para pesquisa da presença de sintomas de insônia, os alunos responderam a um questionário específico para insônia e 38 (76\%) relataram esses sintomas, com predomínio da insônia inicial (36,96\%) e sono não reparador (34,78\%).

Tabela 2. Pontuações do PSQl e ESSE entre os sexos femininos e masculino

\begin{tabular}{llccc}
\hline Sexo & & $\mathbf{n ~ ( 5 0 )}$ & Média & Desvio padrão \\
\hline \multirow{2}{*}{ Masculino } & ESSE (pontuação) & 23 & 8,739 & 2,5975 \\
& PSQl (pontuação) & 23 & 6,043 & 2,1633 \\
\hline \multirow{2}{*}{ Feminino } & ESSE (pontuação) & 27 & 9,000 & 3,1501 \\
& PSQl (pontuação) & 27 & 7,407 & 3,2017 \\
\hline
\end{tabular}

ESE - Escala de Sonolência de Epworth; PSQI - Índice de Qualidade de Sono de Pittsburgh 
De acordo com o resultado da pontuação total do PSQI, 37 (74\%) alunos apresentaram pontuação maior ou igual a 5 pontos (qualidade ruim do sono) e $13(26 \%)$ pontuaram abaixo de 4 (boa qualidade de sono).

Para rastreio de SED, foi utilizada a ESE, em que pontuações acima de 10 foram consideradas portadores de SED. Dos 50 alunos estudados, 16 (32\%) apresentaram critérios de SED.

De acordo com o teste $t$ de Student, ao se comparar os resultados das pontuações de PSQI e ESE entre os gêneros, não foi observado diferença significativa nesses resultados $(p=0,48$ e $p=0,78$, respectivamente).

Tabela 3. Comparação entre os Testes TMT parte A, TMT parte B e DSST entre alunos com e sem SED

\begin{tabular}{l|lcccc}
\hline \multicolumn{1}{l}{ SED } & $\mathbf{n}$ (50) & Mediana & Média & Desvio padrão \\
\hline \multirow{3}{*}{$\begin{array}{l}\text { Presença de } \\
\text { SED }\end{array}$} & TMT parte A & 16 & 27,3400 & 24,6813 & 5,74083 \\
& TMT parte B & 16 & 34,6900 & 38,6288 & 14,41671 \\
& DSST & 16 & 60,50 & 56,250 & 8,0705 \\
\hline \multirow{3}{*}{$\begin{array}{l}\text { Ausência de } \\
\text { SED }\end{array}$} & TMT parte A & 34 & 26,5850 & 27,2315 & 7,18494 \\
& TMT parte B & 34 & 41,6200 & 45,7479 & 18,21613 \\
& DSST & 34 & 58,00 & 55,471 & 7,8672 \\
\hline
\end{tabular}

SED - Sonolência diurna excessiva; TMT - Trail Making Test; DSST - Digit Symbol Substitution Test

Conforme descrito na Tabela 3, ao se aplicar 0 teste $t$ de Student, foi observado que os resultados dos tempos de execução do TMT (partes A e B) foram menores nos alunos com critérios de SED, porém sem significado estatístico $(p=0,2$ e $p=0,16$, para TMT parte A e TMT parte $B$, respectivamente). A pontuação no DSST foi superior nos alunos com SED, mas também não atingiu significância estatística $(p=0,88)$.

Tabela 4. Comparação entre os Testes TMT parte A, TMT parte B e DSST entre alunos com e sem insônia

\begin{tabular}{|c|c|c|c|c|c|}
\hline \multicolumn{2}{|l|}{ Sono } & \multirow{2}{*}{$\frac{\mathbf{n}(\mathbf{4 6})}{38}$} & \multirow{2}{*}{$\begin{array}{c}\text { Mediana } \\
27,3400\end{array}$} & \multirow{2}{*}{$\frac{\text { Média }}{25,5079}$} & \multirow{2}{*}{$\begin{array}{c}\text { Desvio padrão } \\
6,75250\end{array}$} \\
\hline & TMT parte $\mathrm{A}$ & & & & \\
\hline \multirow[t]{3}{*}{ Insônia } & TMT parte $B$ & 38 & 34,6900 & 43,4574 & 19,30900 \\
\hline & DSST & 38 & 60,50 & 56,184 & 7,8115 \\
\hline & TMT parte $\mathrm{A}$ & 8 & 26,5850 & 30,1838 & 6,57939 \\
\hline \multirow[t]{2}{*}{ Sono normal } & TMT parte B & 8 & 41,6200 & 45,8888 & 6,77923 \\
\hline & DSST & 8 & 58,00 & 54,250 & 7,7044 \\
\hline
\end{tabular}

TMT - Trail Making Test; DSST - Digit Symbol Substitution Test

Conforme descrito na Tabela 4, os alunos com critérios para SED apresentaram menor tempo de execução do TMT (partes A e B) e maior pontuação no DSST, porém não atingiu significado estatístico $(p=0,2 ; p=0,13 ; p=69$ para 0 TMT parte $A$, TMT parte B e DSST, respectivamente).

Ao se comparar alunos com boa qualidade de sono (PSQI até 4 pontos) com aqueles com qualidade 
de sono ruim (PSQI com 5 ou mais pontos), aqueles classificados como apresentando qualidade de sono ruim apresentaram tempos de execução do TMT (partes A e B) menores e pontuações no DSST maiores. Contudo, como nos demais testes, não houve diferença estatisticamente significativa $(p=0,09$; $p=0,44 ; p=25$, para o TMT parte A, TMT parte B e DSST, respectivamente).

Tabela 5. Comparação entre os Testes TMT (parte A e B) e DSST entre alunos a partir da pontuação no PSQI

\begin{tabular}{l|lcccc}
\hline \multicolumn{1}{l|}{ PSQI } & $\mathbf{n ~ ( 5 0 )}$ & Mediana & Média & Desvio padrão \\
\hline $\begin{array}{l}\text { Boa qualidade } \\
\text { do sono (PSQI }\end{array}$ & TMT parte A & 13 & 28,7 & 30,0 & 7,6 \\
até 4 pontos) & TMT parte B & 13 & 41,2 & 49 & 23,8 \\
& DSST & 13 & 55,0 & 53,3 & 8,5 \\
\hline \multirow{2}{\text{Qualidaderuim}}{ do sono (PSQI $\leq$} & TMT parte A & 37 & 25,9 & 25,1 & 6,2 \\
5 pontos) & TMT parte B & 37 & 39,0 & 41,5 & 14,2 \\
& DSST & 37 & 58,0 & 56,6 & 7,5 \\
\hline
\end{tabular}

Tabela 6. Comparação entre os Testes TMT (parte A e B) e DSST entre alunos a partir do tempo de sono

\begin{tabular}{l|lccc}
\hline \multicolumn{1}{l}{ HORAS DE SONO } & $\mathbf{n ~ ( 5 0 )}$ & Média & Desvio padrão \\
\hline \multirow{3}{*}{$\begin{array}{l}\text { Menor que 6 horas } \\
\text { de sono }\end{array}$} & TMT parte A & 13 & 25,1933 & 5,64880 \\
& TMT parte B & 13 & 43,2183 & 10,60727 \\
& Digit Symbol & 13 & 54,333 & 8,3267 \\
\hline \multirow{3}{*}{$\begin{array}{l}\text { Maior ou igual a 6 } \\
\text { horas de sono }\end{array}$} & TMT parte A & 37 & 26,7689 & 7,25073 \\
& TMT parte B & 37 & 43,8884 & 19,16229 \\
& Digit Symbol & 37 & 55,973 & 7,7906 \\
\hline
\end{tabular}

TMT - Trail Making Test; DSST - Digit Symbol Substitution Test.

Resultados semelhantes foram observados em alunos que habitualmente têm menos que $6 \mathrm{~h}$ de sono noturno em relação àqueles que relataram dormir pelo menos $6 \mathrm{~h}$ durante a noite. Ou seja, os tempos de execução do TMT (partes A e B) foram menores naqueles que dormem menos, enquanto a pontuação no DSST foi maior nos alunos que dormem pelo menos 6 h durante a noite (Tabela 6). Nessas análises também não foi atingido significância estatística $(p=0,07 ; p=0,82 ; p=34$ para 0 TMT parte $A$, TMT parte B e DSST, respectivamente).

Foi aplicada a correlação linear de Person pela distribuição normal dos dados analisados. Não houve correlação estatisticamente significativa entre os tempos de execução de TMT parte A, TMT parte B e pontuação no DSST com as pontuações do PSQI e ESE e as horas de sono noturno no grupo de alunos de medicina estudados (Tabela 7). 
Tabela 7. Coeficiente de correlação de Pearson ( $r$ ) e seu respectivo nível descritivo $(p)$ dos testes neurocognitivos (TMT parte A e B e DSST) em relação às horas de sono noturno e pontuações nas escalas PSQI e ESE

\begin{tabular}{llccc}
\hline Correlação linear de Pearson & TMT parte A & TMT parte B & DSST \\
\hline \multirow{2}{*}{ ESSE (pontuação) } & Correlação de Person (r) &,- 206 &,- 053 &,- 098 \\
& $p$ & 0,157 & 0,716 & 0,502 \\
\hline \multirow{2}{*}{ PSQI (pontuação) } & Correlação de Person (r) &,- 126 &,- 022 &,- 087 \\
& $p$ & 0,389 & 0,882 & 0,552 \\
\cline { 2 - 5 } & Correlação de Person (r) &,- 128 &,- 064 &, 000 \\
\hline
\end{tabular}

ESE - Escala de Sonolência de Epworth; PSQI - Índice de Qualidade de Sono de Pittsburgh; TMT - Trail Making Test; DSST - Digit Symbol Substitution Test.

\section{DISCUSSÃO}

Estima-se que $30 \%$ a $50 \%$ da população apresentam dificuldades ocasionais para dormir, porém a prevalência de insônia variará de acordo com os critérios para o seu diagnóstico (FORTIERBROCHU; MORIN, 2013). Dependendo dos critérios para diagnóstico de insônia, sua prevalência na população em geral varia de 8 a $38 \%$ (IBRAHIM et al., 2013). Em alguns estudos em que os sintomas de insônia foram estudados sem critérios mais restritivos, foram observadas altas prevalências (de $33 \%$ a $71 \%$ ) (ROCHA; GUERRA; LIMA-COSTA, 2002), (LEGER et al., 2000). No presente estudo com estudantes de medicina, a prevalência de sintomas de insônia foi superior à da população em geral.

Em estudo realizado em 2013 por Tafoya e col. em um grupo de 572 estudantes de medicina, a insônia inicial foi referida por apenas 3,5\% alunos, enquanto as queixas de insônia intermediária $(6,3 \%)$ e terminal $(11,4 \%)$ foram mais frequentes. Neste trabalho não foi estudada a presença do denominado sono não reparador. Essa prevalência foi bem inferior à observada no presente estudo, em que a presença de dificuldade, para se iniciar o sono, foi relatada por $37 \%$ dos alunos, seguida de sono não reparador $(34,8 \%)$, insônia terminal $(10,9 \%)$ e insônia intermediária $(2,2 \%)$.
A amostra de estudantes de medicina analisada neste estudo apresentou uma média de horas dormidas por noite $(6,03 \mathrm{~h})$ inferior à média da população adulta em geral (de sete a nove horas) (POYARES; TUFIK, 2003).

Em um estudo publicado por Cardoso et al., em 2009, foram aplicados os questionários PSQI e ESE a uma amostra de 276 estudantes de medicina revelando que 22,8\% apresentavam qualidade ruim do sono e $51,5 \%$, sonolência excessiva diurna.

Recentemente (MORAES et al., 2013), foram aplicados os mesmos questionários para um grupo de 157 estudantes de medicina. De acordo com o ESE, rastreou-se SED em 36,3\% desses alunos e 44,6 \% foram classificados como apresentando qualidade ruim do sono (PSQI).

Por meio da avaliação da pontuação do PSQI, foi encontrada maior prevalência de qualidade ruim do sono $(74 \%)$ na amostra do presente estudo. Observou-se maior prevalência de SED (32\%) em relação à população em geral (até aproximadamente 18\%) (SLATER; STEIER, 2012), porém não acima da encontrada em alguns trabalhos com estudantes de medicina (CARDOSO et al., 2009) (MORAES et al., 2013).

No presente estudo, os alunos com sintomas de insônia apresentaram qualidade ruim de sono ao PSQI, critérios de SED ao ESE e que referiram ter menos que $6 \mathrm{~h}$ de sono noturno não apresentaram 
diferença significativamente estatística nos resultados dos testes neuropsicológicos aplicados (TMT e DSST).

Após revisão sistemática da literatura, não foram encontrados estudos que analisaram a correlação entre quantidade e qualidade do sono noturno e capacidade cognitiva entre estudantes de medicina.

Em estudo realizado por Fernandez-Mendoza e col, em 2010, foi aplicada uma bateria de testes neuropsicológicos, incluindo o TMT, em um grupo de 1.405 pessoas, divididas entre pacientes com e controles, dependendo da presença de critérios para o diagnóstico de insônia. Não houve diferença estatisticamente significativa das pontuações dos testes entre os grupos, quando pareados, exceto quando passou a se avaliar os pacientes a respeito do tempo médio de sono. A análise da pontuação nos testes evidenciou redução estatisticamente significativa apenas naqueles indivíduos que tinham tempo médio de sono inferior a $6 \mathrm{~h}$, independente do preenchimento de critérios para o diagnóstico de insônia. Aqueles que apresentavam tempo de sono maior, apresentando critérios de insônia ou não, tinham melhores pontuações nos testes neuropsicológicos.

Em uma revisão publicada recentemente (BASTIEN, 2011), foi descrito que o prejuízo cognitivo, relacionado à presença de insônia, é detectado especialmente em testes que analisam atenção, tempo de reação e memória semântica. É descrito também que não há comprovação de interferência da insônia sobre outras funções cognitivas, como habilidades psicomotoras, atenção alternada e memória de trabalho, que são as principais funções estudadas pelo TMT e pelo DSST.

No presente estudo, apesar de os estudantes de medicina com pior quantidade e qualidade de sono noturno, sintomas de insônia e presença de SED apresentarem surpreendentemente melhores resultados nos testes neuropsicológicos aplicados, não houve diferença estatisticamente significativa entre esses grupos de alunos.

\section{CONCLUSÃO}

$\mathrm{Na}$ amostra de estudantes de medicina estudada foram observadas alta prevalência de insônia, qualidade ruim de sono e SED. Diferente da hipótese inicial, em que os alunos de medicina com transtornos de sono poderiam apresentar pior desempenho nos testes neurocognitivos aplicados, os alunos com sintomas de insônia, que dormem menos que $6 \mathrm{~h}$, com pior qualidade de sono e critérios para SED, não apresentaram pior desempenho nos tempos de execução do TMT e na pontuação do DSST. Futuros trabalhos serão necessários para estudar se transtornos do sono se correlacionarão com pior desempenho acadêmico e nos resultados de testes neuropsicológicos que permitam analisar outros domínios da cognição em estudantes de medicina.

\section{REFERÊNCIAS}

BASTIEN, C. H. Insomnia: Neurophysiological and Neuropsychological Approaches. Neuropsychology Review, Nova lorque, v. 21, p. 22-40, 2011.

BERTOLAZI A, N. et al. Validação da escala de sonolência de Epworth em português para uso no Brasil. Jornal Brasileiro de Pneumologia, Brasília, v. 35, n. 9, p. 877-883, 2009.

BUYSSE, D. J. et al. The Pittsburgh Sleep Quality Index: A New Instrument for Psychiatric Practice and Research. Psychiatry Research, San Diego, v. 28, p. 193-213, 1989.

CARDOSO, H. C. et al. Avaliação da qualidade do sono em estudantes de Medicina. Revista Brasileira de Educação Médica, Rio de Janeiro, v. 33, n. 3, p. 349355, fev. 2009.

CARVALHO, T. M. C. S. et al. Qualidade do sono e sonolência diurna entre estudantes universitários de diferentes áreas. Revista Neurociências, São Paulo, v. 21 , n. 3, p. 383-387, 2013.

CHELLAPPA, S. L.; ARAUJO, J. F. Qualidade subjetiva do sono em pacientes com transtorno depressivo. 
Estudos de Psicologia, Natal, v. 12, n. 3, p. 269-274, 2007.

DURMER, J. S.; DINGES, D. F. Neurocognitive consequences of sleep deprivation. Seminars in Neurology, Old Tappan, v. 25, n. 1, p. 117-129, 2005.

FERNANDEZ-MENDOZA, J. et al. Insomnia with objective short sleep duration is associated with deficits in neuropsychological performance: a general population study. Sleep, Darien, v. 10, n. 4. p. 459 465, 2010.

FORTIER-BROCHU, E.; MORIN, C. Cognitive impairments in individuals with insomnia: clinical significance and correlates. Sleep Medicine, v. 14, p. e30, 2013.

GUYTON, A. C.; HALL, J. E. Estados de atividade cerebral: sono, ondas cerebrais, epilepsia, psicoses. In: GUYTON, A. C. Tratado de fisiologia médica. Rio de Janeiro: Elsevier, 2006. p. 739-747.

HERSHNER, S. D.; CHERVIN, R. D. Causes and consequences of sleepiness among college students. Nature and Science of Sleep, Londres, v. 6, 2014. Disponível em <https://www.dovepress.com/causesand-consequences-of-sleepiness-amongcollege-students-peer-reviewed-fulltext-articleNSS > . Acesso em 11 out. 2015.

IBRAHIM, A. K. et al. A systematic review of studies of depression prevalence in university students. Journal of Psychiatric Research, v. 47, n. 3, p. 391-400, 2013.

LEGER, D. et al. Prevalence of insomnia in a survey of 12,778 adults in France. Journal of sleep research, 0xford, v. 9, n. 1, p. 35-42, mar. 2000.

LOPEZ-GÓNGORA, M.; QUEROL, L.; ESCARTÍN, A. A one-year follow-up study of the Symbol Digit Modalities Test (SDMT) and the Paced Auditory Serial Addition Test (PASAT) in relapsing-remitting multiple sclerosis: an appraisal of comparative longitudinal sensitivity. BMC Neurology, Londres, v. 15, p. 40, 2015.
MORAES, C. A. T. et al. Qualidade de sono em estudantes de medicina do método de aprendizado baseado em problemas. Medicina, Ribeirão Preto, v. 46. n. 4, p. 389-397, 2013.

MORIN, C. M.; JARRIN, D. C. Epidemiology of Insomnia. Prevalence, Course, Risk Factors, and Public Health Burden. Sleep Medicine Clinics, Philadelphia, v. 8, p. 281-297, 2013.

$\emptyset$ YANE, N. M. F. et al. Associations between night work and anxiety, depression, insomnia, sleepiness and fatigue in a sample of Norwegian nurses. PLOS ONE, San Francisco, v. 8, n. 8, 2013. Disponível em <http:// journals.plos.org/plosone/article?id=10.1371/journal. pone.0070228 >. Acesso em: 11 out. 2015.

POYARES, D.; TUFIK, S. I Consenso Brasileiro de Insônia: introdução. Hypnos: Revista de Sono, São Paulo, p. 5, 2003.

ROCHA, F. L.; GUERRA, H. L.; LIMA-COSTA, M. F. $F$. Prevalence of insomnia and associated sociodemographic factors in a Brazilian community: the Bambuí study. Sleep Medicine, v. 3, n. 2, p. 121-6, mar. 2002.

ROZENTHAL, M. Avaliação neuropsicológica. In: NARDI, A. E. et al. (Org.) Propsiq. São Paulo: Artmed Panamericana, 2011. p. 95-130.

SLATER, G.; STEIER, J. Excessive daytime sleepiness in sleep disorders. Journal of Thoracic Disease, v. 4, $\mathrm{n}$. 6, p. 608-616, 2012.

TAFOYA, S. A. et al. Dificultades del sueño y síntomas psicológicos en estudiantes de medicina de la Ciudad de México. MEDICINA, Buenos Aires, v. 73, p. 247251, 2013.

TOMBAUGH, T. N. Trail Making Test A and B: Normative data stratified by age and education. Archives of Clinical Neuropsychology, 0xford, v. 19, p. 203-214, 2004.

Recebido em: 18 de outubro de 2015 Aceito em: 08 de abril de 2016 